\title{
Simulation of Near Continuum, Hypersonic Flow Using a Modular Particle-Continuum Method
}

\author{
Timothy R. Deschenes, Iain D. Boyd ${ }^{\dagger}$ \\ Department of Aerospace Engineering, University of Michigan, Ann Arbor, MI, 48109 \\ and \\ Thomas E. Schwartzentruber ${ }^{\ddagger}$ \\ Department of Aerospace Engineering and Mechanics, University of Minnesota, Minneapolis, MN, 55455
}

\begin{abstract}
A modular particle-continuum (MPC) method is used to simulate a low Knudsen number, steady-state hypersonic flow which exhibit small regions of collisional nonequilibrium within a mainly continuum flow field. This method loosely couples an existing direct simulation Monte Carlo (DSMC) code to a Navier-Stokes solver (CFD) while allowing both time-step and cell size to be completely decoupled between each method. Full thermal nonequilibrium (rotational and vibrational) is implemented in both flow modules with compatible relaxation models. The goal of the present investigation is to study the effect of locally rarefied regions on the heat transfer along the after body of a planetary probe body. Previous research suggests that poor agreement in after body heating could be due to locally rarefied regions along the rear surface of blunt bodies in hypersonic flow. Hybrid simulations are compared to full CFD and experimental measurements for high density, hypersonic flow over a sting-mounted planetary probe configuration. By using a hybrid method, the effect of rarefied regions can be examined for a very low Knudsen number case where the computational expense of performing full DSMC calculations is very high due to the four order of magnitude variation in characteristic length and time scales and unnecessary due to the large continuum region. Despite an increase in physical accuracy in the wake region, the initial MPC results have the same level of agreement with experimental measurements as the full CFD solutions. This could be due to insufficient transient time for the MPC simulation and will be explored in the future.
\end{abstract}

\section{Nomenclature}

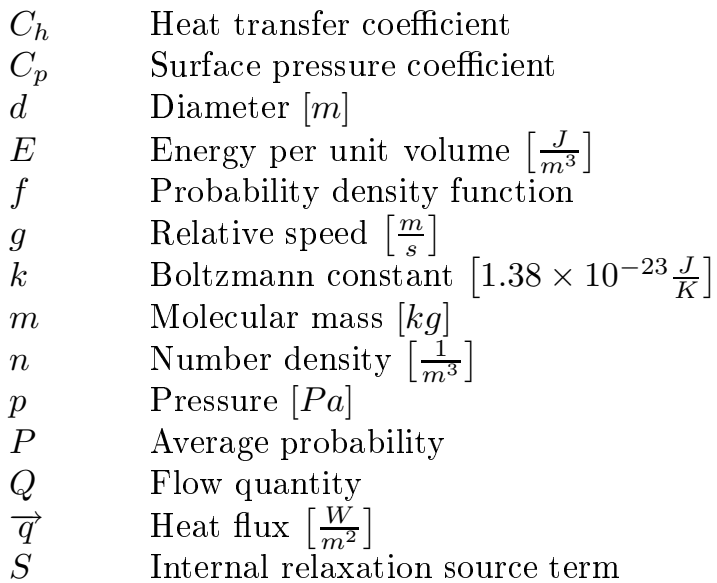

\footnotetext{
*Graduate Student, Student Member AIAA. Email: thytimo@umich.edu.

${ }^{\dagger}$ Professor, Associate Fellow AIAA. Email: iainboyd@umich.edu.

$\ddagger$ Assistant Professor, AIAA Member. Email: schwartz@Ame.umn.edu.
} 
$T \quad$ Temperature $[K]$

$U \quad$ Velocity component in the $\mathrm{x}$ direction $\left[\frac{\mathrm{m}}{\mathrm{s}}\right]$

$V \quad$ Velocity component in the y direction $\left[\frac{S}{s}\right]$

$w \quad$ Source term

Z Collision number

$\eta \quad$ Number of internal degrees of freedom

$\lambda \quad$ Mean free path $[m]$

$\mu \quad$ Coefficient of viscosity $\left[\frac{\mathrm{kg}}{\mathrm{ms}}\right]$, reduced mass $[\mathrm{kg}]$

$\nu \quad$ Mean collision frequency $\frac{1}{s}$

$\Phi \quad$ Subrelaxation weight

$\phi \quad$ Variable probability

$\rho \quad$ Mass density $\left[\frac{\mathrm{kg}}{\mathrm{m}^{3}}\right]$

$\theta \quad$ Characteristic temperature $[K]$

$\sigma \quad$ Reference cross section $\left[\mathrm{m}^{2}\right]$

$\tau \quad$ Relaxation time $[s]$

$\omega \quad$ VHS viscosity temperature exponent

$\begin{array}{ll}\text { Subscripts } \\ \text { CFD } & \text { Computational Fluid Dynamics } \\ D S M C & \text { direct simulation Monte Carlo } \\ M W & \text { Millikan and White } \\ N-W & \text { Near wall } \\ P & \text { Park } \\ \text { ref } & \text { Reference } \\ \text { rot } & \text { Rotational } \\ \text { s } & \text { Species } \\ \text { slip } & \text { Wall slip } \\ \text { tra } & \text { Translational } \\ \text { vib } & \text { Vibrational } \\ \infty & \text { Free stream }\end{array}$

\section{Introduction}

Hypersonic vehicles traveling through the upper atmosphere create a multi-scale flow problem due to the large variation in the characteristic flow length and time scales. The parameter often used to compare these length scales with the vehicle is the Knudsen number which is defined as the ratio of the mean free path $(\lambda)$ to a characteristic length scale $\left(l_{c}\right)$. At very low Knudsen numbers, the flow can be considered very near collisional equilibrium due to the many molecular collisions that occur around the vehicle. For these very near equilibrium flows, the approximations inherent in the formulation of the Navier-Stokes equations are valid and can be readily solved using Computational Fluid Dynamics (CFD). As the Knudsen number increases, the number of molecular collisions occurring around the body decrease and the approximations used to close the Navier-Stokes equations become invalid, and the flow is considered to be rarefied, or in collisional nonequilibrium. Direct simulation Monte Carlo (DSMC) is a computational method that can reproduce the Boltzmann equation which correctly describes the kinetic nature of the flow for these dilute gases. Though valid across all Knudsen numbers, DSMC becomes computationally expensive at very low Knudsen numbers due to cell size and time-step restrictions inherent in the method. Even at very low global Knudsen numbers, there are often regions such as the shock, boundary layer, and wake that exhibit nonequilibrium effects contained in a mainly continuum flow field. Despite being small, the locally rarefied regions can have a large effect on flow results of interest such as the aerodynamics or heat transfer to the body. For these flows, CFD can not be applied to the entire flow field due to the physical inaccuracy, while full DSMC is computationally expensive and unnecessary since the majority of the flow field is continuum.

Hypersonic, low density flow over blunt bodies is one example where neither full CFD nor full DSMC can be applied to the entire flow field. In the fore-body region, the mean free path is sufficiently small enough 
to be solved using CFD, but would require a computationally expensive DSMC simulation. In contrast, the mean free path in the wake is much larger and a kinetic description is required to obtain physically accurate results since the assumptions made in the Navier-Stokes equations are invalid. Because of this, various research in simulating low density wake flows with a hybrid continuum-rarefied flow solvers have been performed over the last 15 years. The first set of results were obtained from "zonally decoupled" CFDDSMC methods. ${ }^{1-4}$ Here, the forebody region is first simulated using CFD. Then, information is extracted along a predetermined interface line and used as inflow information for the rarefied simulation of the wake region. No information is passed back from the rarefied solver to the continuum solver. This restriction often needlessly increases the size of the rarefied domain to include pockets of continuum flow which significantly increases the computational cost of the flow solver. It also forces all interfaces to lie within supersonic regions of the flow due to the inability to pass information back to the continuum solver.

To eliminate the limitations of "zonally decoupled" hybrid flow solvers, numerous coupled hybrid rarefiedcontinuum flow solver have been developed..$^{5-12}$ Here, information is transfered throughout the simulation while both solvers update the solution in their respective domains. This makes two way information transfer possible which allows the continuum-rarefied interface location to be located in subsonic regions of the flow. It also allows the interface location to be dynamically updated based on the current solution. Various choices to determine the final interface location and coupling methods have been proposed and tested. This type of method can maintain the physical accuracy of full DSMC in any rarefied region, while gaining the computational efficiency of CFD in regions that are considered continuum.

For this work, we use the Modular Particle-Continuum (MPC $)^{11,13}$ method, a coupled hybrid CFD-DSMC method, to simulate a very low Knudsen number flow over a sting-mounted planetary probe configuration where the rarefied region is only located in the near wake region. Section 2 will briefly show some previous verification and validation results from tests of the MPC method and list other attempts at applying a coupled hybrid CFD-DSMC solver to the very low Knudsen number flow over a planetary probe configuration. Section 3 will present the MPC method used in this work, including implementation of physical models of interest in each flow module. Section 4 will show a comparison of results derived from the MPC method with full CFD results and experimental measurements, while Sec. 5 lists conclusions and future work.

\section{Previous Research}

One of the most complete, published data sets for hypersonic, blunt body flow with wake measurements is for a sting mounted planetary probe. A series of measurements at multiple facilities in the United States and Europe were conducted in the mid-1990's. ${ }^{14,15}$ The free stream conditions ranged from entirely rarefied to nearly fully continuum. Full DSMC has been applied to all but the very lowest Knudsen number cases. With a four order magnitude variation of length and time scales for the lowest Knudsen number cases, full DSMC would be computationally prohibitive and unnecessary. Previous studies have successfully applied the MPC method to other cases, including higher Knudsen number planetary probe flows, and found the results to be in excellent agreement with both available experimental measurements and full DSMC results. For example, Fig. 1(a) shows a comparison of heat transfer to the planetary probe at a global Knudsen number of 0.002 predicted by full DSMC, full CFD, the MPC method, and experimental measurements that is taken from Ref. 13. The MPC results are in excellent agreement with full DSMC results and both agree with the experimental measurements. In addition, past MPC research of hypersonic flow over other configurations has shown excellent agreement with full DSMC as well. For example, Fig. 1(b) shows the prediction of surface heat transfer from full DSMC, full CFD, and the MPC method for Mach 15 flow over a cylinder with a global Knudsen number of 0.01 which is taken from Ref. 12. Again, the MPC method is in excellent agreement with full DSMC along the entire body even in regions where CFD over predicts the heat transfer by more than a factor of 5 . Based on previous successful reproduction of full DSMC results with the MPC method, this work is the initial attempt at applying the MPC method to a flow to examine a small rarefied region embedded in a large continuum flow field where full DSMC results are infeasable to obtain.

To the authors' knowledge, only one attempt ${ }^{6}$ at applying a coupled hybrid DSMC/Navier-Stokes method to the very near equilibrium cases with a global Knudsen number less than $2 \times 10^{-4}$ has been conducted. Hash found that coupled hybrid results of Mach 10.3 flow of reacting air with a global Knudsen number of $1 \times 10^{-4}$ to be on the same level of agreement with experimental data as his full Navier-Stokes results. He attributed the lack of improved agreement with experimental measurements to an inability to properly 


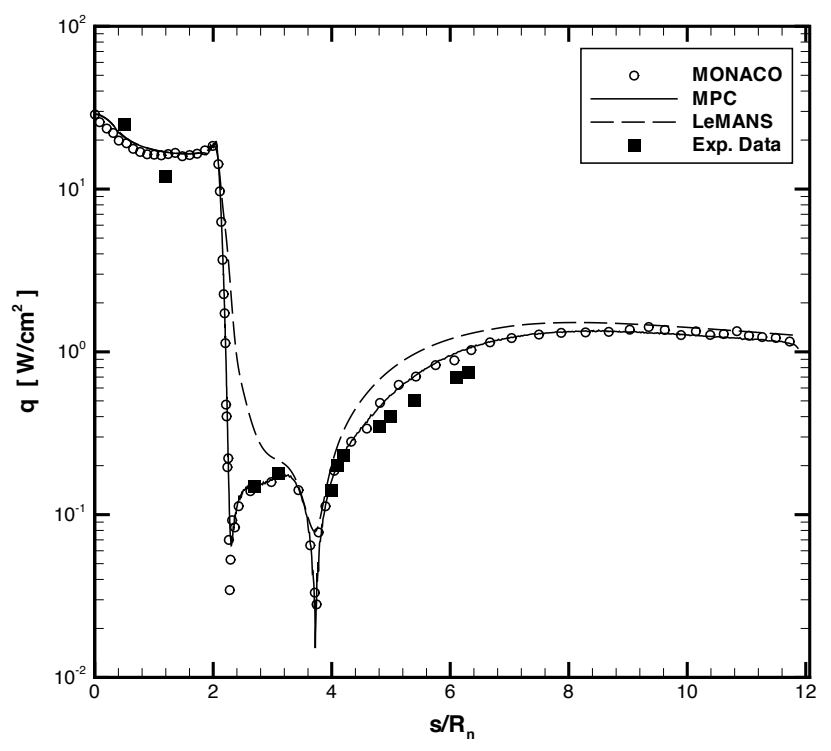

(a) LENS Configuration from Ref. 13.

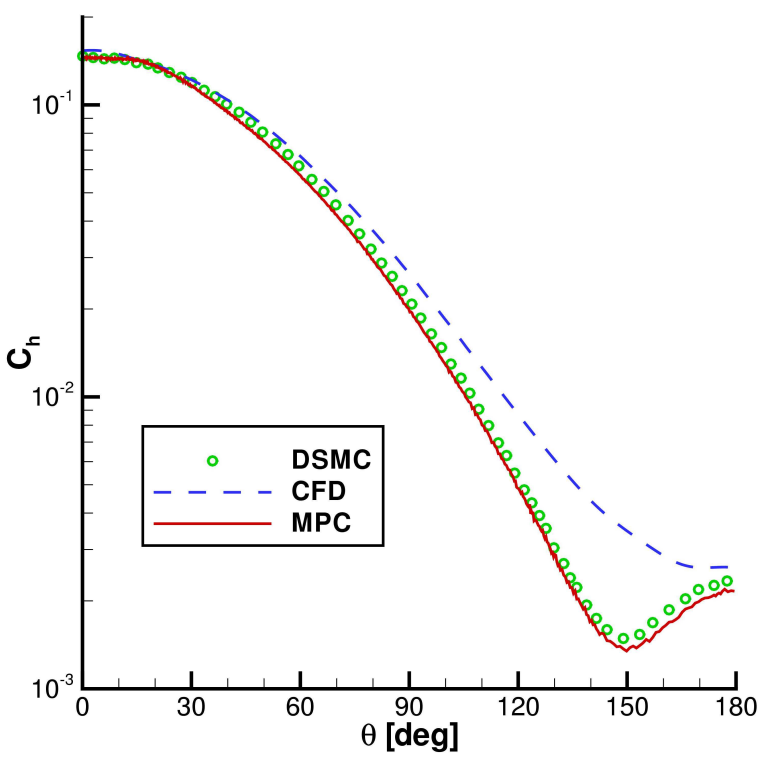

(b) Cylinder configuration from Ref. 12 .

Figure 1. Comparison of heat flux predicted by DSMC, CFD, and the MPC method for various flow geometries and flow conditions.

refine the cells near the cold sting. Based on previous successful verification of the MPC method with full DSMC results, this study will be preliminary work on applying the MPC method to the highest density flow of non-reacting $N_{2}$ over the planetary probe configuration and produce results that contain higher fidelity physics than are available in the Navier-Stokes equations.

\section{The MPC method}

The modular particle-continuum (MPC) method is capable of simulating planar shock waves ${ }^{10}$ and two dimensional and axi-symmetric flows. ${ }^{11,13,16}$ For the continuum regions, it uses LeMANS, while MONACO is used for rarefied regions. Very few modifications are made to each source code which allows the use of state of the art simulation methods that have been previously developed and verified. Instead, the focus is on the hybrid methodology, particularly where the interface between CFD and DSMC is located and when and how information should be transferred between the two modules. The first two subsections will outline the important physical models for the flow of interest employed in each flow solver, while the proceeding subsection summarizes the methodology used in the framework of the MPC method.

\section{III.A. CFD Module}

LeMANS is a laminar, hypersonic code that uses CFD to solve the Navier-Stokes equations that are modified to account for rotational and vibrational nonequilibrium. The Variable Hard Sphere (VHS) viscosity model, as seen in Eqs. 1 and 2, is used to be consistent with the DSMC module. For all simulations presented in this paper, diatomic nitrogen is used with a reference temperature, $T_{r e f}$, of $273 \mathrm{~K}$ and a reference diameter, $d_{\text {ref }}$, of $4.17 \times 10^{-10} \mathrm{~m}$. The power law exponent used is $\omega=0.75$ while $m$ is the molecular mass, $k$ is the Boltzmann constant, and $T_{t r a}$ is the local translational temperature. The inviscid fluxes are solved in LeMANS using a modified version of the Steger-Warming flux vector splitting method. This method is less dissipative outside of the shock which is required to resolve the boundary layer, but switches back to the original form of the Steger-Warming fluxes within the shock. Viscous fluxes are calculated using both values at the cell centers and nodes. For this work, the method uses a point-implicit time-integration method. More 
details about the numerical implementation can be seen in Ref. 17.

$$
\begin{gathered}
\mu=\mu_{\text {ref }}\left(\frac{T_{\text {tra }}}{T_{\text {ref }}}\right)^{\omega} \\
\mu_{\text {ref }}=\frac{15 \sqrt{\pi m k T_{r e f}}}{2 \pi d_{r e f}^{2}(5-2 \omega)(7-2 \omega)}
\end{gathered}
$$

Rotational nonequilibrium is modeled using a separate rotational energy equation as shown in Eq. 3 where $\vec{q}_{\text {rot }}$ is the rotational heat flux given by Fourier's law, $\vec{u}_{d s}$ is the species diffusion velocity given by Fick's law and $\dot{w}_{\text {rot }}$ is the rotational energy source term. Equation 4 shows that the rotational energy source term can be split into two parts, one to model the translational-rotational relaxation process and one to account for loss and gain of rotational energy due to chemical reactions. For this work, chemistry is neglected, so the second term is zero.

$$
\begin{aligned}
\frac{\partial E_{r o t}}{\partial t}+\nabla \cdot\left(E_{r o t} \vec{u}\right) & =-\nabla \cdot\left(\vec{q}_{r o t}\right)-\nabla \cdot \sum_{s}\left(\rho_{s} e_{r o t, s} \vec{u}_{d s}\right)+\dot{w}_{r o t} \\
\dot{w}_{r o t} & =\sum_{s}\left(S_{r o t, s}^{t r a-r o t}+\dot{w}_{s} e_{r o t, s}\right)
\end{aligned}
$$

The rotation-translation relaxation process is modelled using Eq. 5 where $e_{\text {rot,s }}^{*}$ is the specific rotational energy based on the translational temperature, $e_{r o t, s}$ is the current specific rotational energy, $Z_{\text {rot,s }}$ is the rotational collision number, and $\tau_{\text {coll,s }}$ is the mean collision time. The rotational collision number shown in Eq. 6, is derived from Parker. ${ }^{18}$ The mean collision frequency, which is the inverse of the mean collision time, for the VHS model given by Bird ${ }^{19}$ is shown in Eq. 7.

$$
\begin{gathered}
S_{\text {rot }, s}^{\text {tra-rot }}=\rho_{s} \frac{e_{\text {rot }, s}^{*}-e_{\text {rot }, s}}{\tau_{\text {rot }, s}}=\rho_{s} \frac{e_{\text {rot }, s}^{*}-e_{\text {rot }, s}}{Z_{\text {rot }, s} \tau_{\text {coll }}} \\
Z_{r o t, s}=\frac{Z_{r o t, s}^{\infty}}{1+\frac{\pi^{\frac{3}{2}}}{2}\left(\frac{T_{s}^{*}}{T_{\text {tra }}}\right)^{\frac{1}{2}}+\left(\frac{\pi^{2}}{4}+\pi\right)\left(\frac{T_{s}^{*}}{T_{\text {tra }}}\right)} \\
\nu_{s}=\frac{1}{\tau_{\text {coll }, s}}=\sum_{i}\left(n_{i} d_{r e f, s, i}^{2}\left(\frac{8 \pi k T_{r e f, i}}{\mu_{s, i}^{*}}\right)^{\frac{1}{2}}\left(\frac{T_{t r a}}{T_{r e f, i}}\right)^{1-\omega_{i}}\right)
\end{gathered}
$$

In a similar manner to rotational nonequilibrium, LeMANS also includes a separate energy equation, as seen in Eq. 8, to model vibrational nonequilibrium. Here, $\vec{q}_{v i b}$ is the vibrational heat flux given by Fourier's law, $\vec{u}_{d s}$ is the species diffusion velocity given by Fick's law, and $\dot{w}_{v i b}$ is the vibrational source term. Again, the vibrational source term can be split into two parts, as seen in Eq. 9, which are used to model the vibrational-translational relaxation process and the change of total vibrational energy due to chemistry. For this work, chemistry is neglected so the second source is zero.

$$
\begin{aligned}
\frac{\partial E_{v i b}}{\partial t}+\nabla \cdot\left(E_{v i b} \vec{u}\right) & =-\nabla \cdot\left(\vec{q}_{v i b}\right)-\nabla \cdot \sum_{s}\left(\rho_{s} e_{v i b, s} \vec{u}_{d s}\right)+\dot{w}_{v i b} \\
\dot{w}_{v i b} & =\sum_{s}\left(S_{v i b, s}^{t r a-v i b}+\dot{w}_{s} e_{v i b, s}\right)
\end{aligned}
$$

The vibration-translation relaxation process can be model using Eq. 10, where $e_{v i b, s}^{*}$ is the specific vibrational energy evaluated at the translational temperature and $\tau_{v i b, s}$ is the species vibration-translation relaxation time. The relaxation time can be modeled using the summation the of Landau-Teller approximation, shown in Eq. 11, and Park's phenomenological high temperature correction, shown in Eq. 12. Combining the two, Eq. 13 shows the total vibrational-translational relaxation time used in the CFD module.

$$
S_{v i b, s}^{t r a-v i b}=\rho_{s} \frac{e_{v i b, s}^{*}-e_{v i b, s}}{\tau_{v i b, s}}
$$




$$
\begin{gathered}
\tau_{M W}=\frac{1}{p} \exp \left[A_{s}\left(T_{t r a}^{-\frac{1}{3}}-B_{s}\right)-18.42\right] \\
\tau_{P}=\frac{\sqrt{\frac{\pi m_{s}}{8 k T_{t r a}}}}{\sigma_{s} n} \\
\tau_{v i b, s}^{C F D}=\tau_{M W}+\tau_{P}
\end{gathered}
$$

\section{III.B. DSMC Module}

MONACO is a general, cell-based implementation of the DSMC method capable of simulating 2-D, 3-D, or axially symmetric flows. Modules that model rotational, vibrational, or chemical nonequilibrium of an arbitrary number of species are also available. The VHS collision model that replicates the macroscopic viscosity-temperature dependence under equilibrium conditions which is shown in Eq. 1 is used.

Rotational relaxation is modeled using the phenomenological variable rotational energy exchange probability model of Boyd,${ }^{20}$ that is derived from Parker. ${ }^{18}$ This formulation uses a probability of a rotationally inelastic collision, $\phi_{r o t, s}\left(E_{c}\right)$, that reproduces the macroscopic relaxation time by satisfying Eq. 14 where $E_{c}$ is the total collisional energy between the two particles and $f\left(E_{c}\right)$ is the collisional energy probability distribution function. Lumpkin et al. found that the definitions of continuum and particle relaxation times differ by a factor given by Eq. 15 where $\eta_{\text {int,tra }}$ is the number of rotational degrees of freedom based on the translational temperature and $\omega$ is the VHS temperature exponent. Previous analysis has shown excellent agreement between CFD and DSMC for modeling rotational relaxation. ${ }^{12}$

$$
\begin{gathered}
\bar{P}_{\text {rot }, s}=\frac{1}{Z_{\text {rot }, s}}=\frac{1}{\nu_{s} \tau_{\text {rot }, s}}=\int_{0}^{\infty} \phi_{\text {rot }, s}\left(E_{c}\right) f\left(E_{c}\right) d\left(E_{c}\right) \\
\tau_{\text {rot }}^{D S M C}=\frac{\tau_{\text {rot }}^{C F D}}{1+\frac{\eta_{r o t, t r a}}{4-2 \omega}}
\end{gathered}
$$

Vibration-translation relaxation is modeled using a phenomenological cell-temperature based probability model. This method applies an average probability in each cell using Eq. 16 where $\tau_{v i b, s}^{D S M C}$ is the DSMC vibrational relaxation time and $\nu_{s}$ is the equilibrium VHS collision frequency calculated using Eq. 7 . The vibrational relaxation time used in DSMC can be calculated by applying the Gimelshein correction to the continuum vibrational relaxation time seen in Eq. 17, where $\tau_{v i b}^{C F D}$ is the vibrational relaxation time used in CFD calculated at the local, DSMC cell-based translational temperature, $\eta_{v i b, t r a}$ is the number of vibrational degrees of freedom based on the translational temperature, $\omega$ is the VHS viscosity exponent, and $\theta_{v i b}$ is the vibrational characteristic temperature. ${ }^{21}$ This correction resolves the differences in the definition of the relaxation time between DSMC and CFD for quantized energy distributions, such as the harmonic oscillator approximation used in MONACO and further improves on the Lumpkin correction factor that was originally derived for continuous energy distribution functions.

$$
\begin{gathered}
\bar{P}_{v i b}=\frac{1}{\tau_{v i b, s}^{D S M C} \nu_{s}} \\
\tau_{v i b}^{D S M C}=\frac{\tau_{v i b}^{C F D}}{\frac{1+\frac{1}{2} \eta_{v i b, t r a}^{2} \exp \left[\frac{\theta_{v i b}}{T_{t r a}}\right]}{4-2 \omega}}
\end{gathered}
$$

\section{III.C. Hybrid Implementation}

Both the accuracy and efficiency of a hybrid DSMC-CFD method depend strongly on proper placement of the interface location. For physical accuracy, the interface location must be located within regions that can be considered in collisional equilibrium, where the velocity distribution is only slightly perturbed from equilibrium, and the Navier-Stokes equations are valid. The efficiency of the hybrid method requires the interface between CFD and DSMC to be located near the edge of the collisional equilibrium region. The MPC method uses a gradient-length Knudsen number first proposed by Boyd et al. ${ }^{22,23}$ Equation 18 shows the gradient-length Knudsen number where $\lambda$ is the local mean free path and $Q$ is some flow quantity of 
interest. For this work, gradient-length Knudsen numbers based on density, velocity magnitude, translational temperature, and rotational temperature are used.

$$
K n_{G L-Q}=\lambda\left|\frac{\nabla Q}{Q}\right|
$$

Previous research has shown for hypersonic reentry problems ${ }^{16,24}$ and 1-D normal shock waves ${ }^{10}$ that regions where the maximum gradient-length Knudsen number remains less than 0.05 display less than a $5 \%$ difference in flow quantities predicted by DSMC and CFD assuming perfect gas physics. Therefore, regions where the maximum gradient-length Knudsen number exceeds 0.05 must be simulated with DSMC to maintain physical accuracy within the solution. Though the shock is inherently a nonequilibrium process, CFD can provide an accurate description of the jump across the bow shock in the fore body of high number density flows with no effect on surface properties. ${ }^{25}$ To improve the efficiency of the hybrid method, continuum breakdown will be ignored in the vicinity of the strong shock. This is achieved by ignoring any continuum breakdown region where the gradient-length Knudsen number based on pressure, $K n_{G L-p}$, exceeds a value of unity.

The modular implementation of the MPC method allows both the DSMC and CFD modules to maintain their own mesh and data structure. Information is transferred between each method using a state-based coupling procedure. The state-based coupling procedure assigns boundary cells on the edge of each module domain and transfers flow information, such as density, velocity components, and temperatures to these cells from corresponding cells of the other module. Figure 2 shows a schematic of how data is transferred between the two modules at an interface location.

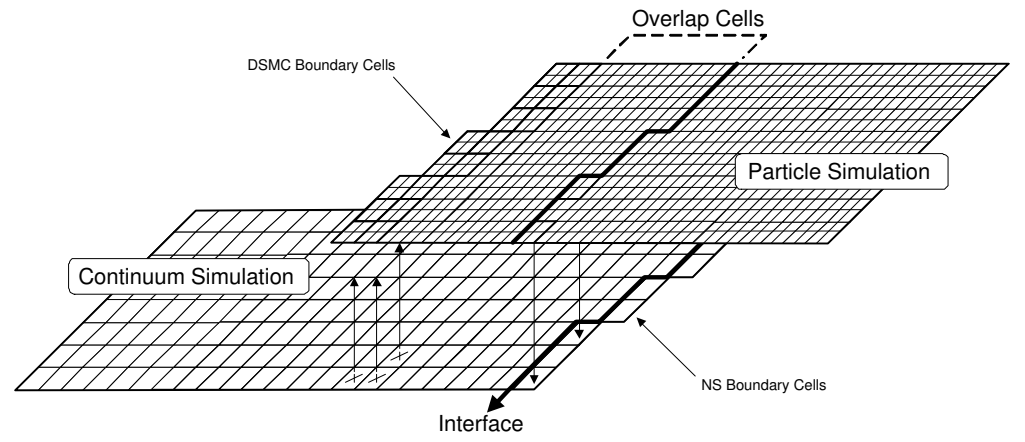

Figure 2. Hybrid particle-continuum coupling procedure. ${ }^{25}$

A DSMC mesh is created from a refined CFD mesh using an estimate of the mean free path from the initial full CFD solution. For this work, DSMC cells are refined to be less than 3 times the mean free path, while a maximum of 25 dynamically created collisional subcells are used to ensure that the mean collision separation between collision pairs is less than a mean free path. Reference 25 provides a detailed outline of the mesh refinement algorithm that is used in this study. Once the DSMC mesh is created, the initial interface location is found using the breakdown parameter shown in Eq. 18. Next, a DSMC buffer region is created. This buffer region is used throughout the unsteady portion of the simulation as the initial CFD solution relaxes to the DSMC result. In order to obtain an accurate DSMC prediction in rarefied regions, the buffer region must be large enough to eliminate any error caused by the initially inaccurate DSMC boundary conditions from the CFD module. For this work, the buffer region is set to 6 CFD cells which ensures that particles experience a sufficient number of collisions before entering the rarefied region. At the beginning of each iteration, DSMC boundary cells are repopulated with particles in a manner consistent with the Chapman-Enskop velocity distribution function based on macroscopic values and gradeints extracted from the local CFD cells. Periodically, the breakdown parameter is re-evaluated to ensure that the entire rarefied region is contained within the DSMC domain. Once the interface location has stopped moving, information from the DSMC region is passed to the CFD edges and the CFD module iterates. Equation 19 shows the subrelaxation average that the MPC method uses to reduce the scatter in information collected from DSMC and applied as boundary conditions to the CFD domain. ${ }^{26}$ This performs a weighted average that applies a small weight to the current sample while applying a larger weight to the previous average. Though it lags the current solution during the unsteady portion of the simulation, agreement improves as the flow reaches steady-state. For this work, a subrelaxation parameter of $\Phi=1 . \times 10^{-3}$ is used. 


$$
\bar{Q}_{j}=(1-\Phi) \bar{Q}_{j-1}+\Phi Q_{j}
$$

For this work, only the near wake region is considered to be rarefied, and the Navier-Stokes equations are physically accurate over most of the domain. This results in very little movement of the interface locations. Figure 3 illustrates this by showing the initial and final CFD-DSMC interface locations for the planetary probe case considered in this work. Once the interface has stopped moving, the MPC method periodically switches between the two modules, while allowing information to be transfered at each module switch as the entire flow field iterates to the steady-state solution. After a user inputted number of iterations, the over-lap regions are removed, interfaces are locked, and DSMC sampling begins.

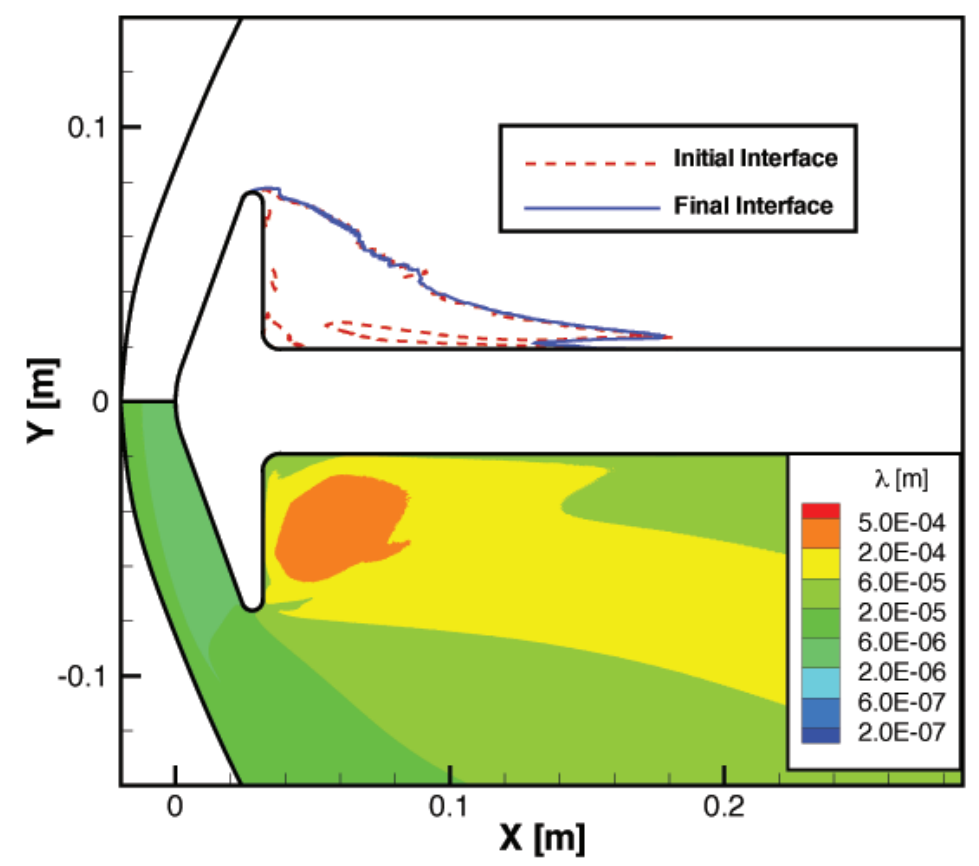

Figure 3. Initial and final interface locations (top) and variation in mean free path (bottom) for Mach 10.3 flow of $N_{2}$ over a planetary probe configuration with a global Knudsen number of $1 \times 10^{-4}$.

\section{Geometry and Flow Conditions}

For this study, numerical simulation of the LENS case D is presented. Figure 4 shows a schematic of the geometry used for the simulations with definitions of the points of interest along the body. The blunted $70^{\circ}$ cone has a nose radius of $3.81 \mathrm{~cm}$ and a shoulder radius of $0.381 \mathrm{~cm}$. The body radius is $7.62 \mathrm{~cm}$ while the sting radius has a value of $1.905 \mathrm{~cm}$. Structured block meshing is used to grid the geometry for the full CFD simulations. Grids are constructed to ensure that the solution was grid independent by doubling the total number of grid points and ensuring that the heat transfer to the body did not change. The final full CFD mesh contained nearly 380,000 computational cells. The corresponding DSMC mesh is created by refining the $\mathrm{CFD}$ mesh in the wake region using the initial CFD solution to estimate the local mean free path. Refinement up to $40 \times 4(160 \mathrm{DSMC}$ cells in $1 \mathrm{CFD}$ cell) occurs along the sting in the near wake region. A total of nearly 450,000 DSMC cells are located in the final rarefied region, with over 9 million simulation particles. Dynamic subcells are employed to ensure that the mean collision separation between particle collisions is less than a mean free path. Due to the limitations of the current, serial implementation of the MPC method, an unsteady total time of $50 \mu \mathrm{s}$ is simulated. Despite initalizing the DSMC particles from the steady-state Navier-Stokes solution, the slow development time in the recirculation region may still have been larger than the simulation time. 


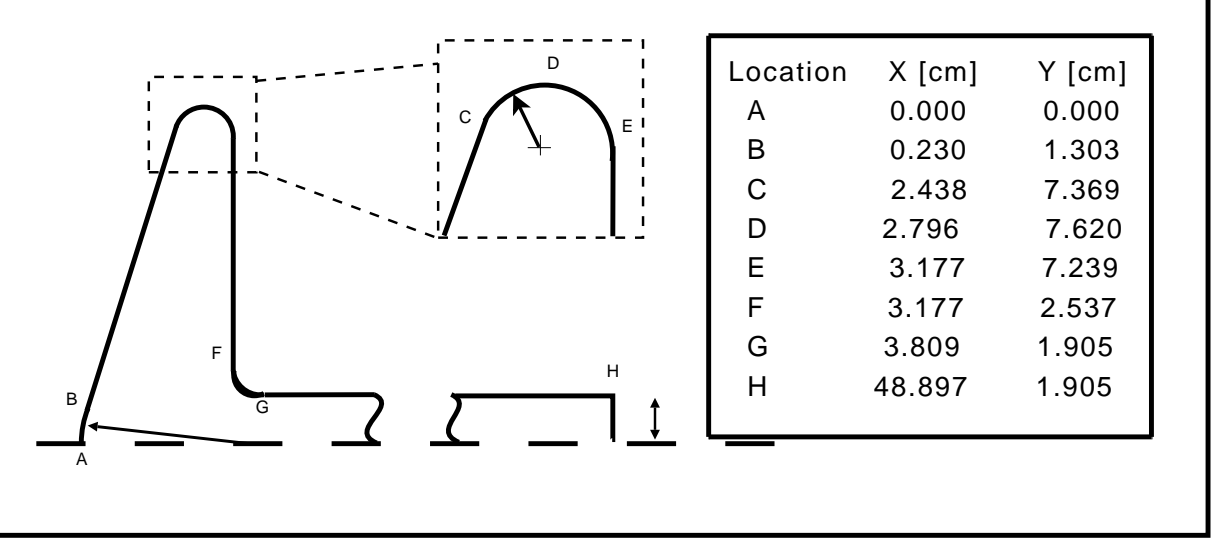

Figure 4. Initial and final interface locations for Mach 10.3 flow of $N_{2}$ over a planetary probe configuration with a global Knudsen number of $1 \times 10^{-4}$.

Flow conditions used in this study are listed in Table 1 which were calculated from Ref. 14. All internal modes are assumed to be in equilibrium with the translational mode and also set to a temperature of $244.8 \mathrm{~K}$. The Navier-Stokes solver employs no slip (temperature or velocity) at the wall boundaries, while DSMC applies full thermal and momentum accommodation for particles colliding with the wall.

Table 1. Experimental flow conditions for the planetary probe.

\begin{tabular}{|c|c|c|c|c|c|c|c|}
\hline Case & $M_{\infty}$ & $U_{\infty}\left[\frac{\mathrm{m}}{\mathrm{s}}\right]$ & $\rho_{\infty}\left[\frac{\mathrm{kg}}{\mathrm{m}^{3}}\right]$ & $T_{\infty}[K]$ & $\lambda_{\infty}[\mathrm{m}]$ & $T_{w}[K]$ & $K n_{\infty}$ \\
\hline \hline LENS-D & 10.31 & $3,287$. & $3.70 \times 10^{-3}$ & 244.8 & $1.6 \times 10^{-5}$ & 294.0 & $1.0 \times 10^{-4}$ \\
\hline
\end{tabular}

\section{Numerical Results}

This section outlines the numerical results derived from both the MPC method and full DSMC. First, flow field properties are presented with a focus on the differences in the near wake region. Next, predicted surface properties are compared with each other and published experimental measurements.

\section{V.A. Flow Field}

For all cases, the rarefied region has a negligible effect on the continuum fore body region. The rarefied region does, however, change the continuum region downstream. Figure 5 compares the horizontal velocity contours predicted between the MPC method and full CFD. In addition, stream lines are post-processed to highlight the differences in the recirculation regions predicted by the two methods. These differences in recirculation regions have a large effect on other near wake flow field properties. In general, the size of the main vortex predicted by CFD and the MPC method are nearly the same, but the MPC method predicts a slightly longer main vortex along the sting mount which could be due to velocity slip along the sting. In addition, the center of the main vortex is slightly higher and further back which is caused by the larger secondary vortices predicted in the MPC solution compared to full CFD. The prediction of these two secondary vortices that are located along the rear face, just below the shoulder and in the sting juncture area are significantly different. First, the MPC method predicts both secondary vortices to be larger than the corresponding CFD simulation. In addition, the MPC method predicts a fourth vortex very near the shoulder that is completely absent in the full CFD results. Also, the MPC method predicts that the streamlines along the edge of each vortex lie much closer to the surface than those predicted by full CFD. These differences also are a result of the slip velocity at the surface that DSMC predicts, while the Navier-Stokes equations predict zero slip. The differences in sizes of secondary vortices has a direct effect on the prediction of the vibrational energy mode contours in the region, and also on the surface pressure and heat flux to the body.

Figure 6 compares the translational temperature contours predicted by full CFD (top) and the MPC method (bottom). The MPC solution predicts a larger region of hotter gas near the wake region of the body. 

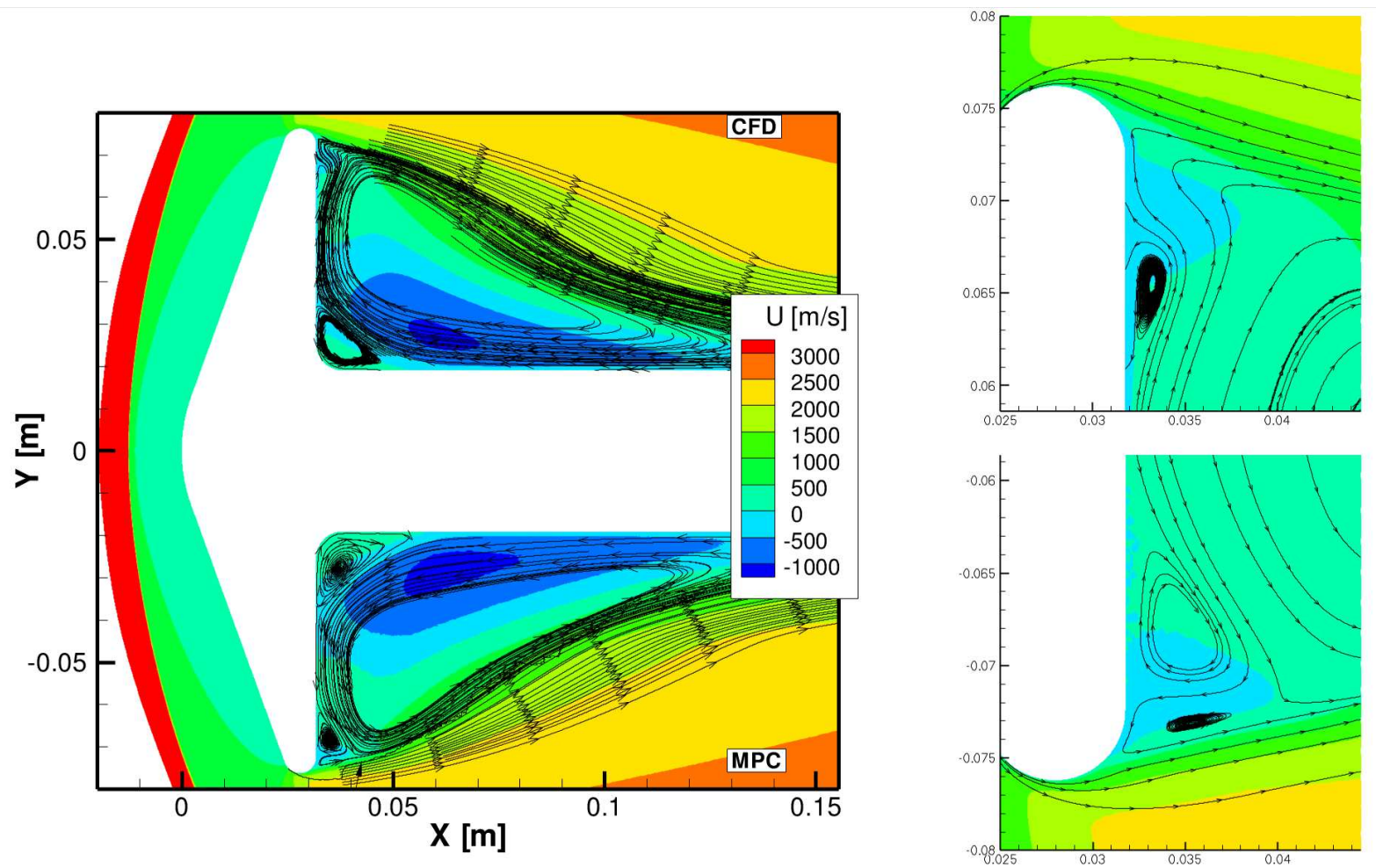

Figure 5. U velocity contours with streamlines of wake vortices predicted by full CFD (top) and the MPC method (bottom) for Mach 10.3 flow of $N_{2}$ over a planetary probe configuration with a global Knudsen number of $1 \times 10^{-4}$.

This could be due to the temperature slip predicted by the MPC method in this region. In addition, the MPC method predicts a cooler temperature along the stream line on the edge of the main vortex, which does not occur in the Navier-Stokes solution. This could be due to the assumptions in the Navier-Stokes equations resulting in a higher collision frequency in this region which has an effect of increased heat conduction than that predicted by the DSMC module used by the MPC method in this region. Finally, the translational temperature predicted along the sting far away from the wake differs in the two solutions, despite both methods employed CFD in this region.

Figure 7 shows the rotational temperature contours predicted by full CFD and the MPC method. Since the rotational energy modes equilibriate with the translational temperature very quickly, the predicted rotational fields are nearly the same as the translational temperature. The one exception is that CFD predicts a slightly smaller region of high rotational temperature $(>1,500 \mathrm{~K})$ gas in the recirculation region, while the MPC method predicts the same size as the translational temperature region. In contrast, Fig. 8 compares the vibrational temperature contours predicted by full CFD and the MPC method. Here, both methods predict the vibrational mode to be very far from equilibrium with the translational mode. In addition, some distinct differences between the two solutions are evident. First, the MPC method actually predicts a faster relaxation process along the streamline that expands very near the shoulder and passes along the edge of the main vortex. This could be in part due to the differences in sizes of the vortex in this region, where the gas is circulated around the vortex more in the MPC solution, but it could also be due to differences in the relaxation rates in the expansion region where the flow transitions very quickly from a large density that can be considered continuum to a rarefied, very low density region in a very short flow time. Finally, the effect of the secondary vortex along the backside of the probe is very evident in the vibrational temperature contour. Here, the larger recirculation of gas predicted by the MPC method allows a vibrationally cool region to develop due to the longer flow time that each fluid element remains in this region. Downstream of the wake, both CFD and the MPC method predict nearly identical vibrational temperature contours across the weak re-compression shock. 


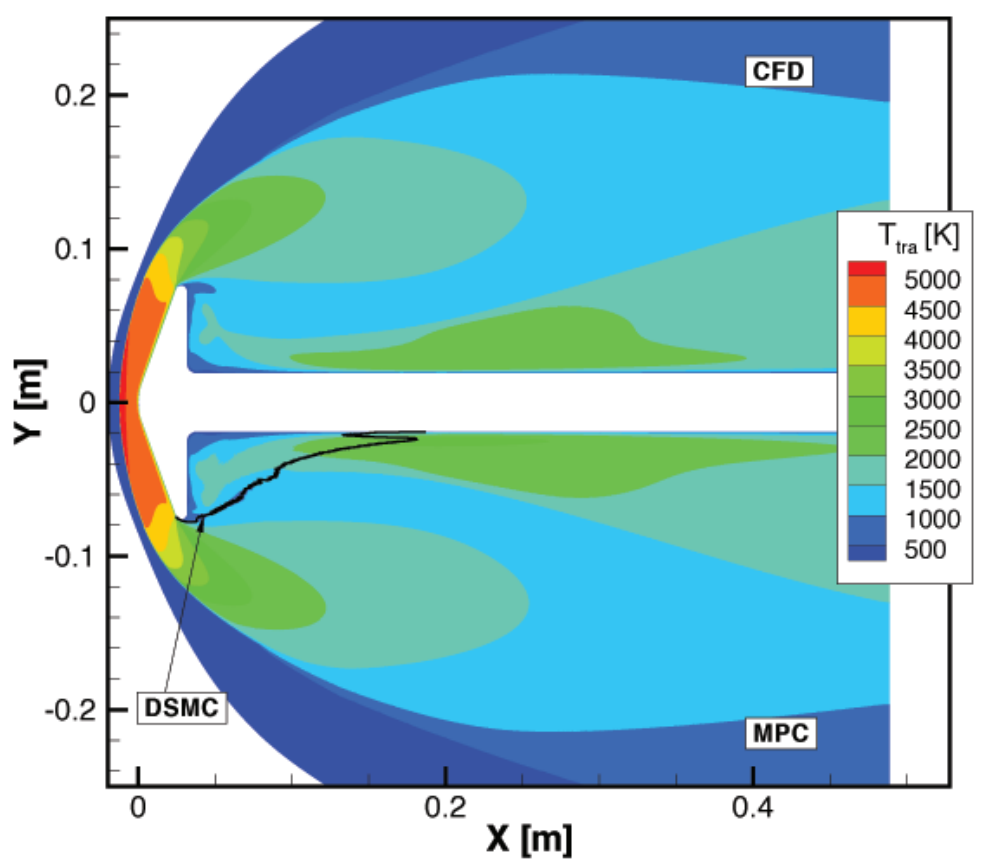

Figure 6. Translational temperature contours predicted by full CFD (top) and the MPC method (bottom) for Mach 10.3 flow of $N_{2}$ over a planetary probe configuration with a global Knudsen number of $1 \times 10^{-4}$.

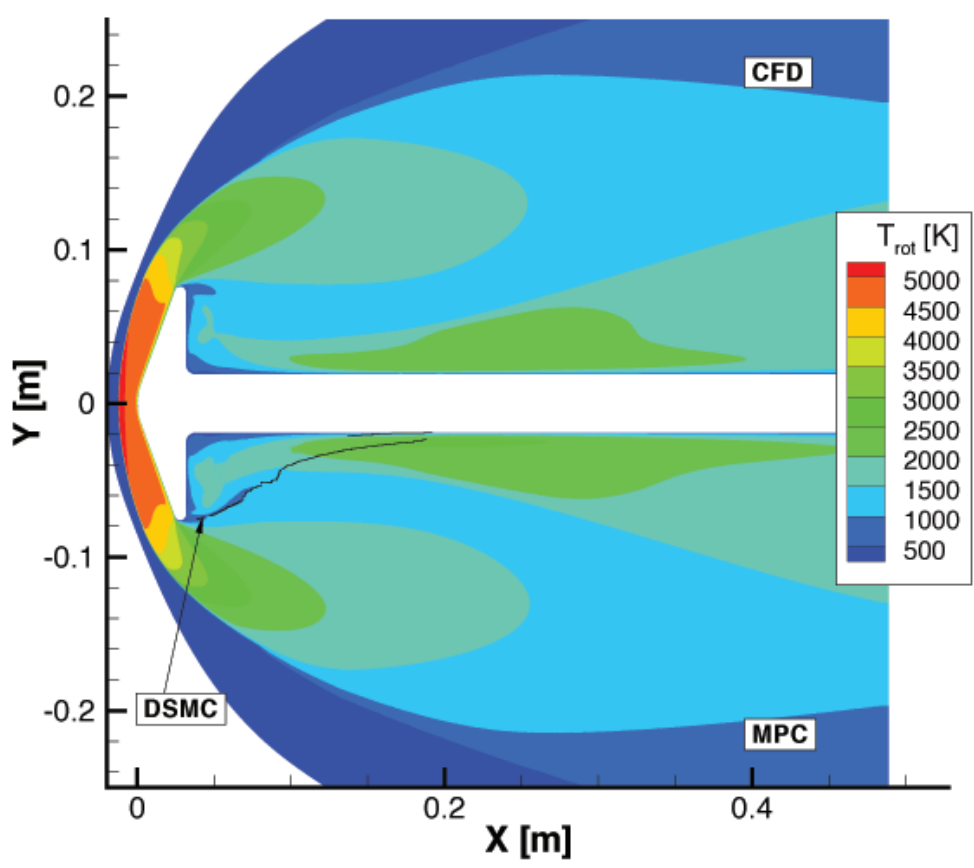

Figure 7. Rotational temperature contours predicted by full CFD (top) and the MPC method (bottom) for Mach 10.3 flow of $N_{2}$ over a planetary probe configuration with a global Knudsen number of $1 \times 10^{-4}$.

\section{1 of 16}




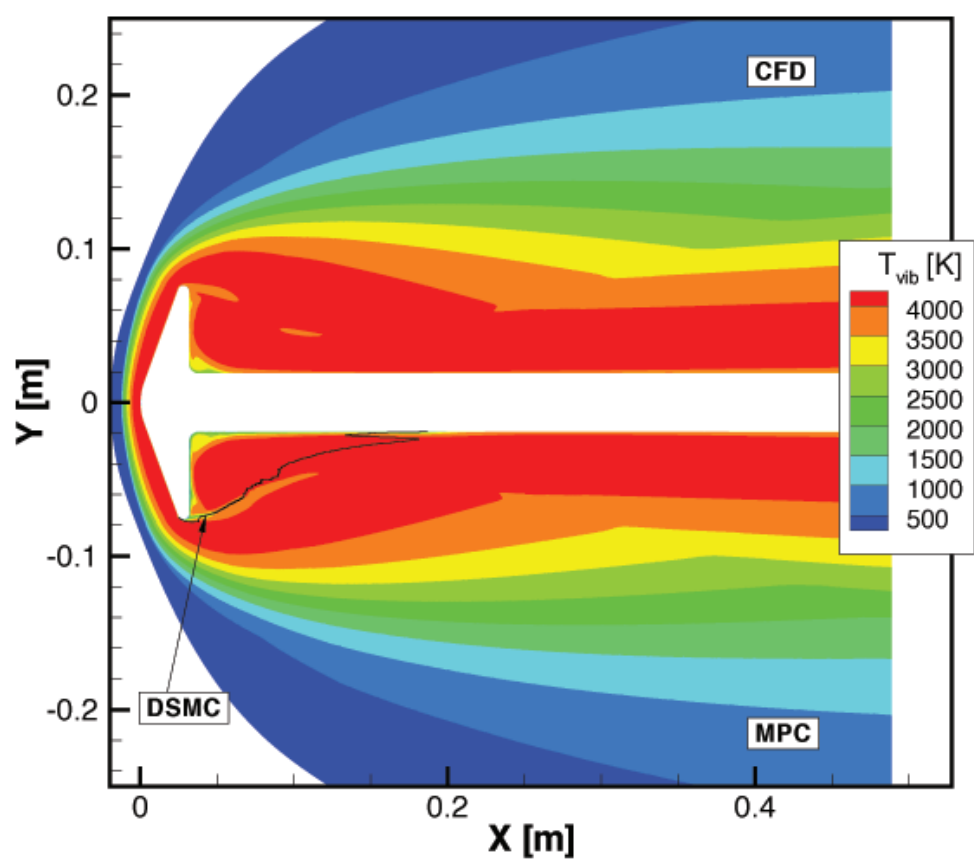

Figure 8. Vibrational temperature contours predicted by full CFD (top) and the MPC method (bottom) for Mach 10.3 flow of $N_{2}$ over a planetary probe configuration with a global Knudsen number of $1 \times 10^{-4}$.

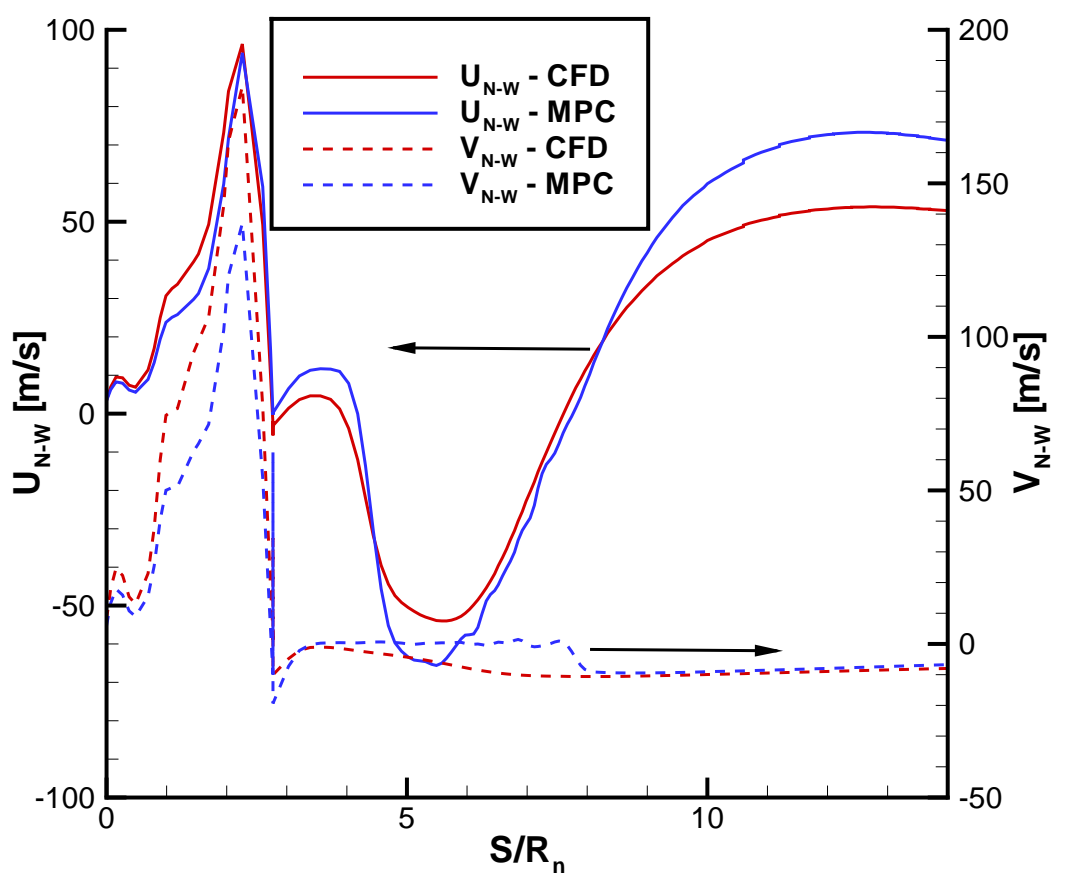

Figure 9. Comparison of velocity near the surface predicted by the MPC method and full CFD for Mach 10.3 flow of $N_{2}$ over a planetary probe configuration with a global Knudsen number of $1 \times 10^{-4}$. 


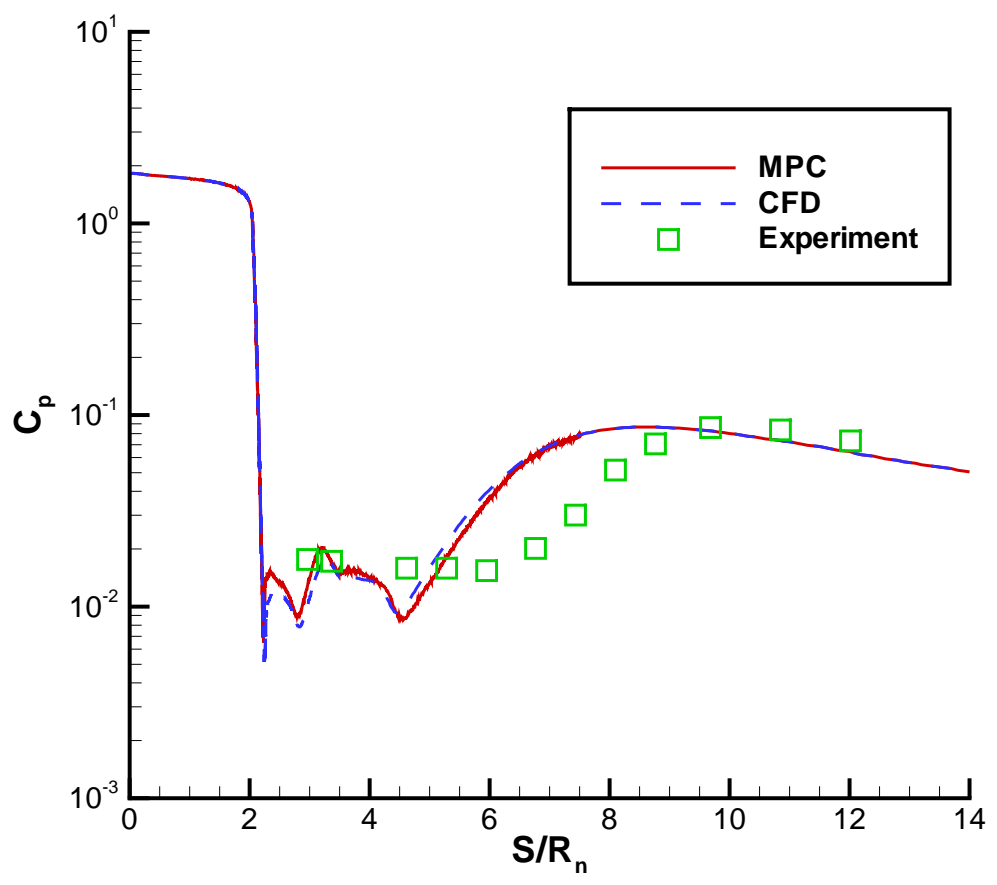

Figure 10. Comparison of surface pressure predicted by the MPC method and full CFD with experimental measurements for Mach 10.3 flow of $N_{2}$ over a planetary probe configuration with a global Knudsen number of $1 \times 10^{-4}$.

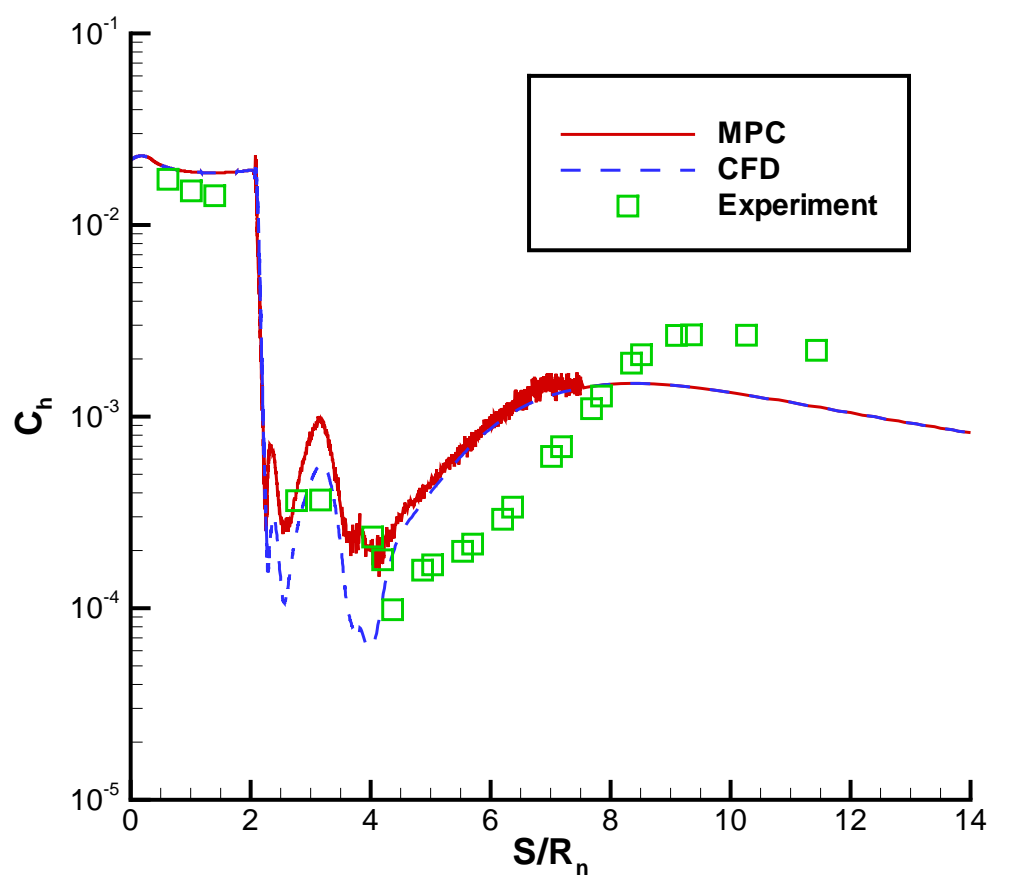

Figure 11. Comparison of heat flux to the body predicted by the MPC method and full CFD with experimental measurements for Mach 10.3 flow of $N_{2}$ over a planetary probe configuration with a global Knudsen number of $1 \times 10^{-4}$. 


\section{V.B. Surface Properties}

In addition to comparison of flow field features predicted by full CFD and the MPC method, surface properties predicted by the two methods can be compared with available experimental measurements. Figure 9 shows the velocity components $5 \mu \mathrm{m}$ away from the wall surface predicted by full CFD and the MPC method. As the flow expands around the shoulder $\left(\frac{S}{R_{n}} \approx 2\right)$, the near-wall velocities predicted by full CFD and the MPC method start to deviate. In addition, the velocities predicted at the sting juncture are qualitatively in agreement, but the MPC method predicts much larger magnitudes. This has a direct effect on the differences in sizes of the wake vortices shown in the previous subsection. Figure 10 compares the coefficient of pressure, defined by Eq. 20, where $p$ is the surface pressure, $p_{\infty}$ is the free stream static pressure, $\rho_{\infty}$ is the free stream density, and $V_{\infty}$ is the free stream velocity. First, the forebody predictions are in excellent agreement with each other which is also evident in the flow field property comparison. Next, despite differences in the flow field properties in the near wake region, there is very little difference in the surface pressure predicted by full CFD and the MPC method. Only very small differences are seen along the sting juncture and sting surface. Both methods showed the same level of agreement with the experimental measurements where both are qualitatively in agreement along the back face, the very near wake, and the far sting region, but neither match the pressure measurements in the mid sting region.

Figure 11 compares the coefficient of surface heating, which is defined by Eq. 21 where $q$ is the heat transfer to the body, between the MPC solution, CFD solution, and experimental measurements. Again, the heat transfer predicted by the two methods is in excellent agreement in the fore body region where the mean free path is very small, and the flow is far into the continuum regime. There is a slight over prediction in heat transfer in the fore body for both methods compared to the experimental measurements. This could be partially due to some neglected physical model, such as finite rate chemistry. In the wake region, the heat transfer predicted by CFD and the MPC method, while qualitatively following the same trends, show differences in the magnitude of heat transfer. First, the larger recirculation zones has a direct effect on the surface heating predicted, with a larger separation between impingement points along the rear face $\left(\frac{S}{R_{n}} \approx 2.07\right)$. Both prediction methods have about the same level of agreement with the experimental measurements along the rear face. In addition, the largest difference in predicted heat transfer between full CFD and the MPC method occurs in the sting juncture area, where the MPC method predicts a heat transfer that is a factor of 8 higher than the full CFD prediction. Again, neither method is in noticably better agreement with the experimental measurements. Along the sting, CFD and the MPC method are in excellent agreement despite differences in the simulation methods used over a large region, which shows that this area of the flow is very near equilibrium and possibly could be simulated with CFD instead of DSMC in the MPC method. Despite the excellent agreement of simulation methods, neither are quantitatively in agreement with the experimental measurements along the far sting region. The differences in surface property predictions with measurements could be due to the boundary layer transitioning from laminar to turbulent after the re-compression region. The authors of the experimental measurements also mention the possibility of a turbulent boundary layer developing during the low density experimental cases or that the experimental test time was too short for the wake to fully reach steady-state. ${ }^{14,15}$ In addition, the MPC simulation may require a longer than $50 \mu s$ unsteady time for the near wake region to reach the steady-state. Even though the DSMC particles were intialized using the Navier-Stokes solution which was simulated for longer than the experimental run time, the slow recirculation regions present in the wake may not had enough time to relax from the continuum solution to the rarefied solution in $50 \mu \mathrm{s}$ of simulation time. Holden et al. found that the heat flux reached a peak and then decreased to the steady-state value ${ }^{14}$ which indicates that too short of an unsteady period before sampling could account for the MPC method predicting higher heat flux in the wake.

$$
\begin{aligned}
C_{p} & =\frac{p-p_{\infty}}{\frac{1}{2} \rho_{\infty} V_{\infty}^{2}} \\
C_{h} & =\frac{q}{\frac{1}{2} \rho_{\infty} V_{\infty}^{3}}
\end{aligned}
$$

\section{Conclusions and Future Work}

Simulation of very low Knudsen number, hypersonic flow with continuum and hybrid methods are presented and compared with available, published experimental measurements. Only a small region in the near 
wake region is detected as rarefied in the MPC method and the DSMC method is applied to this region, while using CFD everywhere else. Due to the very low Knudsen number and over a four order of magnitude variation in length and time scales, it is estimated that full DSMC results would require a factor of $100 \times$ more computational time and memory than the MPC method.

Differences in the predicted flow properties in the near wake region with each method are outlined. In addition, predicted surface pressure and heat transfer are compared with experimental data. Both methods predict nearly identical surface pressure and are in good agreement with measurements. Outside of the near wake region (rear face and sting-juncture), both CFD and the MPC method predict heat transfer that is in very good agreement with each other. In the near wake region, significant differences in the magnitude of heat flux predicted by full CFD and the MPC method are evident, but neither are in better agreement with experimental data.

Though initial MPC predictions do not improve on the full CFD predictions agreement with experimental measurements, the current computational limitations of the serial implementation of the method could have an effect by severly limiting the length of time during the unsteady portion of the MPC simulation. With a parallel code, a longer unsteady time can be simulated without requiring an extremely long wall clock time by taking advantage of parallel computing clusters. With a longer simulation time, other rarefied flow structures that have longer development times could evolve which could impact the agreement with available experimental measurements. In addition to parallelization, further study of a breakdown parameter, particularly with strong internal relaxation processes present, could improve the efficiency of the MPC method by only applying the DSMC method in regions where it is required.

\section{Acknowledgments}

The authors gratefully acknowledge funding from NASA Grant NCC3-989 and from the AFRL Collaborative Center in Aeronautical Sciences. The third author also acknowledges support from the Air Force Office of Scientific Research (AFOSR) under Grant No. FA9550-04-1-0341. The views and conclusions contained herein are those of the authors and should not be interpreted as necessarily representing the official policies or endorsements, either expressed or implied, of the AFOSR or the U.S. Government. Finally, the authors thank Matthew MacLean for providing experimental measurements.

\section{References}

${ }^{1}$ Glass, C. E. and Gnoffo, P. A., "Comparison of a 3-D CFD-DSMC Soultion Methodology With a Wind Tunnel Experiment," Tech. Rep. TM-2002-211777, NASA, August 2002.

${ }^{2}$ Glass, C. E. and Gnoffo, P. A., "A 3-D Coupled CFD-DSMC Solution Method With Application to the Mars Sample Return Orbiter," Tech. Rep. TM-2000-210322, NASA, July 2000.

${ }^{3}$ Wilmoth, R. G., Mitcheltree, R. A., Moss, J. N., and K., D. V., "Zonally Decoupled Direct Simulation Monte Carlo Solutions of Hypersonic Blunt-Body Flows," Journal of Spacecraft and Rockets, Vol. 31, No. 6, 1994, pp. 971-979.

${ }^{4}$ Hash, D. B. and Hassan, H. A., "A Decoupled DSMC/Navier-Stokes Analysis of a Transitional Flow Experiment," Collection of Technical Papers - 34th AIAA Aerospace Sciences Meeting and Exhibit, Vol. 1, Reno, NV, 1996.

${ }^{5}$ Wardswoth, D. C. and Erwin, D. A., "Two-dimensional hybrid continuum/particle simulation approach for rarefied flows," , No. AIAA paper 1992-2975, 1992.

${ }^{6}$ Hash, D. B., A Hybrid Direct Simulation Monte Carlo / Navier-Stokes Flow Solver, Ph.D. thesis, North Carolina State University, 1996.

${ }^{7}$ Roveda, R., B., G. D., and Varghese, P. L., "Hybrid Euler/Particle Approach for Continuum/Rarefied Flows," Journal of Spacecraft and Rockets, Vol. 35, No. 3, 1998, pp. 258-265.

${ }^{8}$ Wang, W. L. and Boyd, I. D., "Hybrid DSMC-CFD Simulations of Hypersonic Flow Over Sharp and Blunted Bodies," , No. AIAA 2003-3644, 2003

${ }^{9}$ Lian, Y. Y., Wu, J. S., Cheng, G., and Koomullil, R., "Development of a parallel hybrid method for the DSMC and NS solver." , No. AIAA 2005-435, 2005.

${ }^{10}$ Schwartzentruber, T. E. and Boyd, I. D., "A hybrid particle-continuum method applied to shock waves," Journal of Computational Physics, Vol. 215, No. 2, July 2006, pp. 402-416.

${ }^{11}$ Schwartzentruber, T. E., Scalabrin, L. C., and Boyd, I. D., "A modular particle-continuum numerical method for hypersonic non-equilibrium gas flows," Journal of Computational Physics, Vol. 225, No. 1, July 2007, pp. 1159-1174.

${ }^{12}$ Deschenes, T. R., Holman, T. D., Boyd, I. D., and Schwartzentruber, T. E., "Analysis of Internal Energy Transfer Withing a Modular Particle-Continuum Method," Vol. AIAA 2009-1213, 2009.

${ }^{13}$ Schwartzentruber, Thomas E.and Scalabrin, L. C. and Boyd, I. D., "Multiscale Particle-Continuum Simulations of Low Knudsen Number Hypersonic Flow Over a Planetary Probe," Journal of Spacecraft and Rockets, Vol. 45, No. 6, 2008, pp. 11961206. 
${ }^{14}$ Holden, M. S., Harvey, J. K., Boyd, I. D., George, J., and Horvath, T. J., "Experimental and Computational Studies of the Flow Over a Sting Mounted Planetary Probe," AIAA-1997-768.

${ }^{15}$ Holden, M. S. and Wadhams, T. P., "A Review of Experimental Studies For DSMC and Navier-Stokes Code Validation in Laminar Regions of Shock/Shock and Shock/Boundary Layer Interaction Including Real Gas Effects in Hypervelocity Flows," , No. AIAA 2003-3641, 2003.

${ }^{16}$ Schwartzentruber, T. E., Scalabrin, L. C., and Boyd, I. D., "Hybrid particle-continuum simulations of nonequilibrium hypersonic blunt-body flowfields," Journal of Thermophysics and Heat Transfer, Vol. 22, No. 1, 2008, pp. 29-37.

${ }^{17}$ Scalabrin, L. C. and Boyd, I. D., "Development of an Unstructured Navier-Stokes Solver for Hypersonic Nonequilibrium Aerothermodynamics," AIAA-2005-5203.

${ }^{18}$ Parker, J. G., "Rotational and Vibrational Relaxation in Diatomic Gases," Phys. Fluids, Vol. 2, No. 4, July 1959, pp. 449-462.

${ }^{19}$ Bird, G. A., Molecular Gas Dynamics and the Direct Simulation of Gas Flows, Clarendon Press, 1994.

${ }^{20}$ Boyd, I. D., "Analysis of rotational nonequilibrium in standing shock waves of nitrogen," AIAA Journal, Vol. 28, No. 11, 1990, pp. 1997-1999.

${ }^{21}$ Gimelshein, N. E., Gimelshein, S. F., and Levin, D. A., "Vibrational Relaxation Rates in the Direct Simulation Monte Carlo Method," Physics of Fluids, Vol. 14, No. 12, 2002.

${ }^{22}$ Boyd, I. D., Chen, G., and Candler, G. V., "Predicting failure of the continuum fluid equations in transitional hypersonic flows," Phys. Fluids, Vol. 7, No. 1, Jan. 1995, pp. 210-219.

${ }^{23}$ Wang, W.-L. and Boyd, I. D., "Predicting continuum breakdown in hypersonic viscous flows," Phys. Fluids, Vol. 15, No. 1, Jan. 2003, pp. 91-100.

${ }^{24}$ Schwartzentruber, T. E., Scalabrin, L. C., and Boyd, I. D., "Investigation of Continuum Breakdown in Hypersonic Flows using a Hybrid Particle-Continuum Algorithm," AIAA-2008-4108.

${ }^{25}$ Schwartzentruber, T. E., Scalabrin, L. C., and Boyd, I. D., "Modular implementation of a hybrid DSMC-NS algorithm for hypersonic non-equilibrium flows," AIAA-2007-613.

${ }^{26}$ Sun, Q. and Boyd, I. D., "Evaluation of Macroscopic Properties in the Direct Simulation Method," Journal of Thermophysics and Heat Transfer, Vol. 19, No. 3, 2005, pp. 329-335. 